\title{
Magnetic Domain Structure and Crystal Orientation Revealed by a Forescatter Detector and Electron Backscatter Diffraction.
}

\author{
Matthew Gallaugher ${ }^{1}$, Nicolas Brodusch ${ }^{1}$, Raynald Gauvin ${ }^{1}$, and Richard R. Chromik ${ }^{1}$ \\ ${ }^{1}$ Department of Mining and Materials Engineering, McGill University, Montreal, Quebec, Canada.
}

Imaging magnetic domains for soft magnetic materials using scanning electron microscopy (SEM) typically requires a specially modified setup. These modifications can include higher beam acceleration voltages, modifications of the sample and detector geometry, and/or specialized detectors to detect the spin polarization of secondary electrons [1,2]. In this research, a method is presented for imaging magnetic domains with type II magnetic contrast using a forescatter detector (FSD) attached to a commercial electron backscatter diffraction (EBSD) camera, with a resolution limit of approximately 200-300 $\mathrm{nm}$. In addition to revealing the magnetic contrast, the EBSD system was used to provide complementary crystal orientation information, which helped to interpret the direction of alignment and fine structure of the magnetic domains. The analysis method was demonstrated on a non-oriented electrical steel (NOES) sample; a material typically used in the motor core of electric vehicle drivetrains.

The width and complexity of the surface magnetic domain structure with no applied magnetic field is related to the magnetostatic energy, which is chiefly determined by the angle $\beta$ between the nearest magnetic easy axis and the surface of the sample [3]. The magnetic easy axis is a crystallographic direction that requires less energy to magnetize $(<100>$ for NOES). As a result, magnetic domains tend to align themselves in these directions [3]. As $\beta$ increases, it produces an increase in the magnetostatic energy, which decreases the domain width and can also increase the complexity of the domain structure. Magnetic domain images were captured at a beam voltage of $30 \mathrm{kV}$ and a sample tilt of $70^{\circ}$ tilt using the bottom two diodes on the FSD, and related to an EBSD map collected in the same experimental conditions. The EBSD map data was also exported, and MATLAB code was used to calculate $\beta$ for each pixel, similar to a calculation performed on a different type of electrical steel [4].

Magnetic domains were clearly visible with this technique, and were found to be in alignment with the closest easy axis to the surface (Figure 1). Domains decreased in width and increased in complexity as $\beta$ increased. Images taken at higher magnification, as shown in Figure 2, were used to evaluate the resolution limits for the technique. In addition to the magnetic domain contrast, domain wall contrast was also observed in some cases (e.g., Figure 2). This technique was shown to be effective at exploring magnetic domain structure and its relationships to crystal orientation using an EBSD system installed on a standard SEM.

\section{References:}

[1] D. Newbury et al, “Advanced Scanning Electron Microscopy and X-Ray Microanalysis”, (Kluwer Academic / Plenum Publishers, NY), (1986), pp. 147-179.

[2] D. J. Fathers et al, Physica Status Solidi (A) Applied Research 20 (2) (1973), pp. 535-544.

[3] Chikazumi, S., "Physics of Ferromagnetism", (Oxford University Press Inc., NY), (2010), pp. 249-256; 439-445.

[4] N. Bernier et al, Micron 54-55 (2013), pp. 43-51.

[5] K. M. Podurets and S. S. Shilstein, Physica B: Condensed Matter 297 (1-4) (2001), pp. 263-267. 

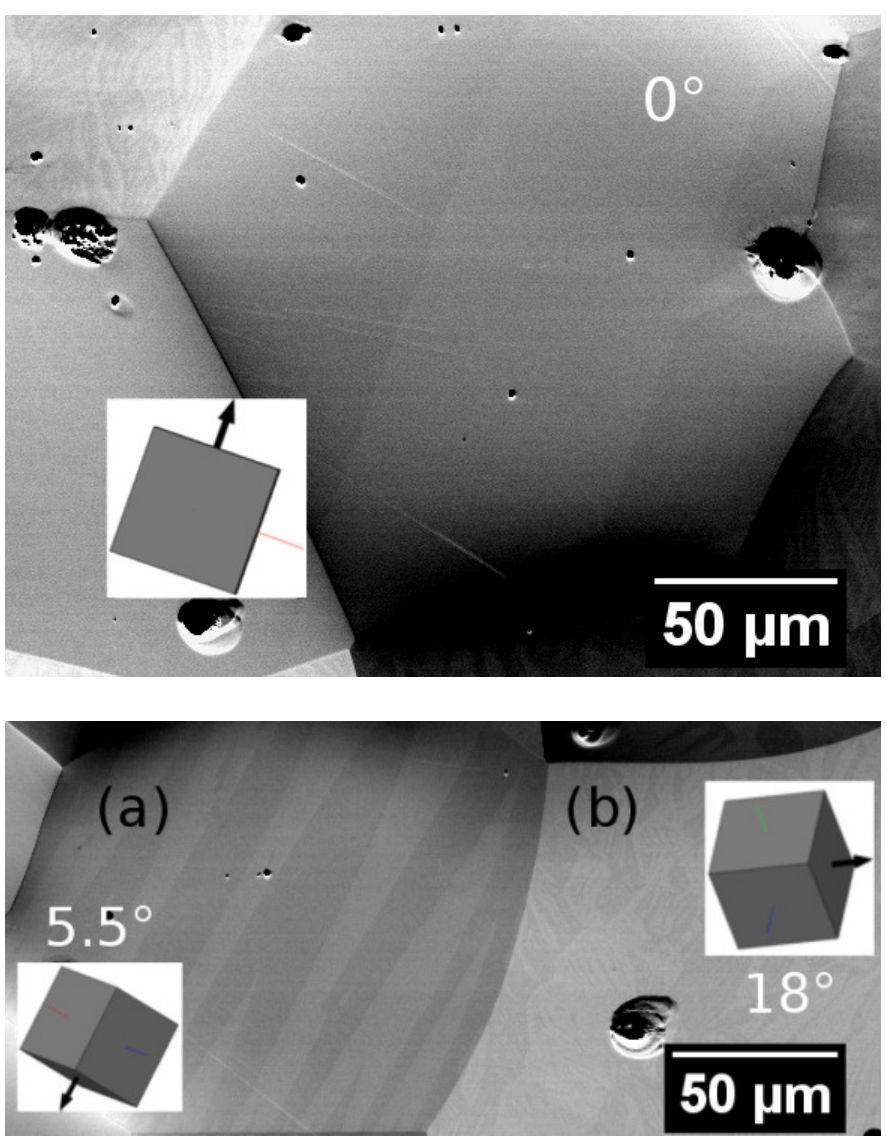

Figure 1. Forescatter detector images showing the magnetic domain structure, and how it related to the crystal orientation (inset crystal image), and the $\beta$ angle (white number in images). As $\beta$ increased, the increase in magnetostatic energy caused the width of the magnetic domain structure to decrease. The black arrow in the inset crystal images identifies the closest easy axis to the surface.
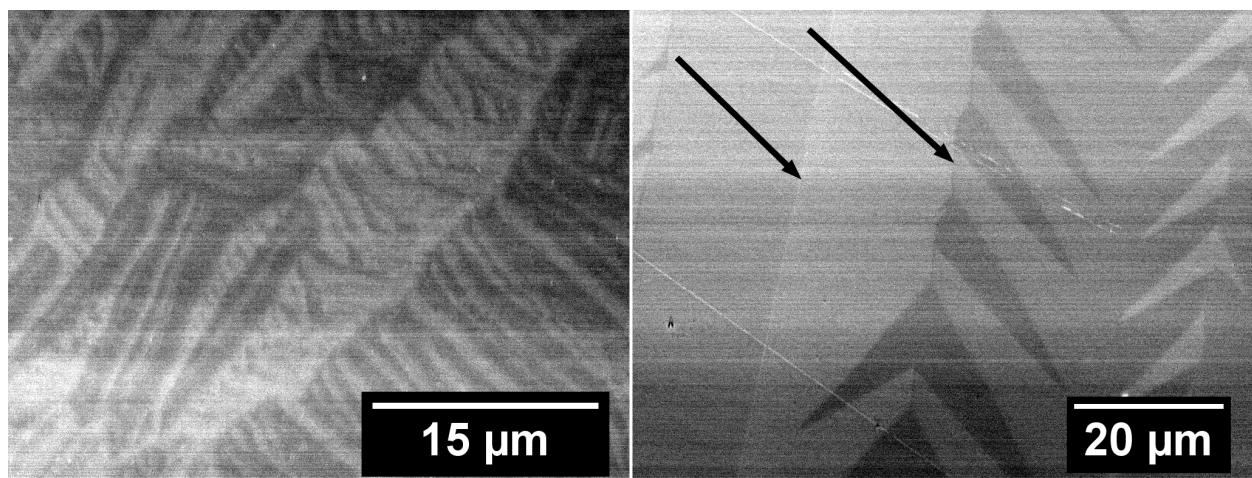

Figure 2. Magnetic domain fine structure as revealed by higher magnification forescatter detector images. From the image on the left, the spatial resolution was evaluated to be $250-300 \mathrm{~nm}$. The image on the right revealed domain wall contrast (light and dark lines in the locations indicated by the arrows). From literature, this type of domain wall for this material is approximately $200 \mathrm{~nm}$ in width [5]. 